\title{
CFL Conditions for Finite Integration Methods on Triangular Meshes
}

\author{
$\operatorname{AUTHOR}(\mathrm{S})$ :
}

Matsuo, Tetsuji; Mifune, Takeshi

\section{CITATION:}

Matsuo, Tetsuji ... [et al]. CFL Conditions for Finite Integration Methods on Triangular Meshes. IEEE TRANSACTIONS ON MAGNETICS 2009, 45(3): 1348-1351

\section{ISSUE DATE:}

2009-03

URL:

http://hdl.handle.net/2433/109812

\section{RIGHT:}

(c) 2009 IEEE. Personal use of this material is permitted. However, permission to reprint/republish this material for advertising or promotional purposes or for creating new collective works for resale or redistribution to servers or lists, or to reuse any copyrighted component of this work in other works must be obtained from the IEEE. 


\title{
CFL Conditions for Finite Integration Methods on Triangular Meshes
}

\author{
Tetsuji Matsuo and Takeshi Mifune \\ Department of Electrical Engineering, Kyoto University, Kyoto 615-8510, Japan
}

In this paper, the CFL conditions of several 2-D finite integration (FI) methods on regular triangular meshes are derived. A piecewise uniform element allows a time step that is twice as large as that for the Whitney element.

Index Terms-Electromagnetic wave, finite integration (FI) technique, piecewise uniform element, stability, Whitney element.

\section{INTRODUCTION}

$\mathbf{F}$ INITE INTEGRATION (FI) methods [1]-[4] enable the use of unstructured meshes in electromagnetic-wave analysis. Several methods to construct permittivity and reluctivity matrices have been proposed [3]-[6] because they greatly affect the efficiency and accuracy of FI methods. The resultant efficiency depends on the computational cost of permittivity matrix inversion and on the Courant condition or the Courant-Friedrichs-Lewy (CFL) condition, which gives the maximum time step for stable computation [7].

Previous works have derived the CFL condition for nonorthogonal mesh [8]-[10]. However, the CFL criterion is not explicitly deduced from the permittivity and reluctivity matrices. Accordingly, although several conditionally stable FI methods have been proposed, their CFL criteria are not obtained explicitly.

This paper derives CFL conditions for several 2-D FI methods using triangular meshes, where the Whitney basis functions [3] and piecewise uniform basis functions [4] are used to construct the permittivity matrix.

\section{CFL CONDITION}

The time stepping of the FI method is written as the following:

$$
\begin{aligned}
\boldsymbol{e}^{k+1} & =\boldsymbol{e}^{k}+\Delta t \boldsymbol{\varepsilon}^{-1} \boldsymbol{C}^{T} \boldsymbol{\nu} \boldsymbol{b}^{k+1 / 2} \\
\boldsymbol{b}^{k+3 / 2} & =\boldsymbol{b}^{k+1 / 2}-\Delta t \boldsymbol{C} \boldsymbol{e}^{k+1}
\end{aligned}
$$

Therein, $\boldsymbol{e}$ denotes the vector of line integrals of the electric field along the edges of primal mesh, $\boldsymbol{b}$ is the vector of magnetic fluxes across the faces of primal mesh, $\boldsymbol{C}$ is the curl matrix (the incidence matrix between faces and edges) for primal mesh, $\Delta t$ is a time step, $\boldsymbol{\varepsilon}$ is the (global) permittivity matrix, and $\boldsymbol{\nu}$ is the (global) reluctivity matrix.

From (1) and (2), the following recursion formula for $\boldsymbol{b}$ is obtained:

$$
\boldsymbol{b}^{k+3 / 2}-\left\{2 \boldsymbol{I}-(\Delta t)^{2} \boldsymbol{\Gamma}\right\} \boldsymbol{b}^{k+1 / 2}+\boldsymbol{b}^{k-1 / 2}=0
$$

Manuscript received October 07, 2008. Current version published February 19, 2009. Corresponding author: T. Matsuo (e-mail: tmastuo@kuee.kyoto-u.ac. jp).

Digital Object Identifier 10.1109/TMAG.2009.2012622

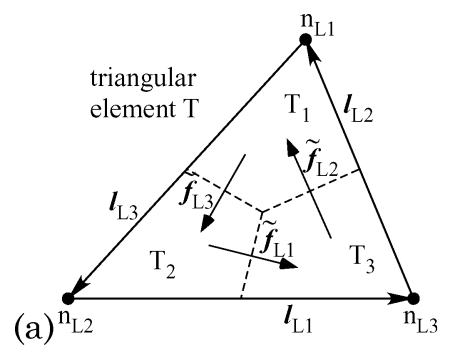

(b)

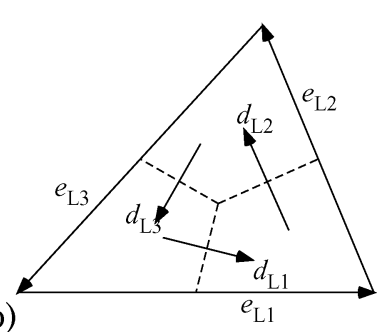

Fig. 1. Triangle element.

where $\boldsymbol{I}$ is a unit matrix and $\boldsymbol{\Gamma}$ is given by

$$
\Gamma=C \varepsilon^{-1} C^{T} \nu .
$$

The matrices $\boldsymbol{\varepsilon}$ and $\boldsymbol{\nu}$ should be positive-definite symmetric for the FI scheme to be conditionally stable [9], [10].

For $\boldsymbol{b}$ to be bounded for $k \rightarrow \infty$, the CFL condition is derived as [10]

$$
\Delta t \leq \frac{2}{\sqrt{\gamma_{\max }}}
$$

where $\gamma_{\max }$ is the maximum eigenvalue of $\Gamma$.

\section{FI Methods For TRANSVERSE Electric (TE) WAVE COMPUTATION ON TRIANGULAR MESH}

\section{A. Local Permittivity Matrix}

This paper discusses the CFL condition for the 2-D FI method using triangular primal mesh with barycentric dual mesh. Fig. 1(a) shows a triangle element $T$ used for FI analysis of a TE wave, where the solid segments $l_{L i}(i=1,2,3)$ are edges of primal mesh (subscript " $L$ " means "local"). The dashed segments $\tilde{f}_{L i}$ denote faces of dual mesh within $T$, which have a height of unit length along the perpendicular direction. The permittivity $\varepsilon$ and permeability $\mu$ are assumed to be uniform within each of the triangular elements.

The line integrals of the electric field along the primal edges are denoted by $e_{L i}(i=1,2,3)$, while the electric fluxes across the dual faces within $T$ are denoted by $d_{L i}$ [see Fig. 1(b)]. The relation between $e_{L i}$ and $d_{L i}$ is written as

$$
d_{L i}=\sum_{j=1}^{3} \varepsilon_{L i j} e_{L j}
$$

where

$$
\boldsymbol{\varepsilon}_{L}=\left\{\varepsilon_{L i j}\right\}, \quad i, j=1,2,3 .
$$


is the local permittivity matrix.

Several methods have been proposed to construct $\varepsilon_{L}$.

The Whitney basis functions $\boldsymbol{w}_{i}(i=1,2,3)$ are written as

$$
\boldsymbol{w}_{i}=\lambda_{j} \nabla \lambda_{k}-\lambda_{k} \nabla \lambda_{j}
$$

where $\lambda_{i}$ are barycentric coordinates corresponding to node $n_{L i}$ and $\{i, j, k\}$ is a cyclic permutation of $\{1,2,3\}$. They give the local permittivity matrix $\boldsymbol{\varepsilon}_{L}^{W}[3]$ as

$$
\varepsilon_{L i j}^{W}=\int_{T} \varepsilon \boldsymbol{w}_{i} \cdot w_{j} d S .
$$

The piecewise uniform basis functions $\boldsymbol{u}_{i}(i=1,2,3)$ [4] are written as

$$
\boldsymbol{u}_{i}= \begin{cases}0, & \text { (in } \left.T_{i}\right) \\ \nabla \lambda_{k}, & \text { (in } \left.T_{j}\right) \\ -\nabla \lambda_{j}, & \text { (in } \left.T_{k}\right)\end{cases}
$$

where $T_{i}$ is a subdomain within $T$ as shown in Fig. 1(a) and $\{i, j, k\}$ is a cyclic permutation of $\{1,2,3\}$. They give the local permittivity matrix $\varepsilon_{L}^{P U}$ as

$$
\begin{aligned}
\boldsymbol{\varepsilon}_{L i j}^{P U} & =\int_{T} \varepsilon \boldsymbol{u}_{i} \cdot \boldsymbol{u}_{j} d S \\
& =\frac{\varepsilon S}{3}\left\{-\nabla \lambda_{i} \cdot \nabla \lambda_{j}+\delta_{i j} \sum_{k=1}^{3}\left(\nabla \lambda_{k}\right)^{2}\right\}
\end{aligned}
$$

where $\delta_{i j}$ is Kronecker's delta.

Both $\varepsilon_{L}^{W}$ and $\varepsilon_{L}^{P U}$ have an eigenvector $(1,1,1)$ of which eigenvalues are $\beta$ and $4 \beta$, respectively, where

$$
\beta=\frac{\varepsilon S}{12} \sum_{i=1}^{3}\left(\nabla \lambda_{i}\right)^{2}=\frac{\varepsilon}{48 S} \sum_{i=1}^{3} l_{i}^{2}
$$

(see Appendix). Therein, $S$ is the element area and $l_{i}=\left|\boldsymbol{l}_{L i}\right|$. The corresponding eigenspace is denoted by $E^{\text {curl }}$.

The orthogonal complementary space of $E^{\text {curl }}$ is denoted by $E^{\text {div }}$, which consists of the (local) electrostatic field $\left(e_{1}+e_{2}+\right.$ $\left.e_{3}=0\right)$. Both the Whitney basis functions and piecewise uniform basis functions give a uniform electric field $\boldsymbol{E}$ when $e_{1}+$ $e_{2}+e_{3}=0$. It is known [4] that for a uniform electric field, $\varepsilon_{L i j}^{W}$ and $\varepsilon_{L i j}^{P U}$ yield the same $d_{L i}$ given by

$$
d_{L i}=E \cdot \tilde{\boldsymbol{f}}_{L i} \text {. }
$$

The subspace $E^{\text {div }}$ affects some eigenvalues of $\boldsymbol{\Gamma}$ because $e_{1}+$ $e_{2}+e_{3}=0$ means only that the electric field is uniform within the element and does not mean curl $\boldsymbol{E}=0$ globally. However, it is expected that $E^{\text {curl }}$ is more important than $E^{\text {div }}$ for the CFL condition.

Reference [3] proposed a diagonalized local permittivity ma$\operatorname{trix} \varepsilon_{L}^{D}$ given as

$$
\varepsilon_{L}^{D}=-\varepsilon S \operatorname{diag}\left(\nabla \lambda_{2} \cdot \nabla \lambda_{3}, \nabla \lambda_{3} \cdot \nabla \lambda_{1}, \nabla \lambda_{1} \cdot \nabla \lambda_{2}\right) .
$$

\section{B. Global Permittivity Matrix}

The global permittivity matrix $\varepsilon$ is constructed by assembling $\varepsilon_{L}$.

For simplicity, the regular triangular mesh as depicted in Fig. 2 is used with periodic boundary conditions along both
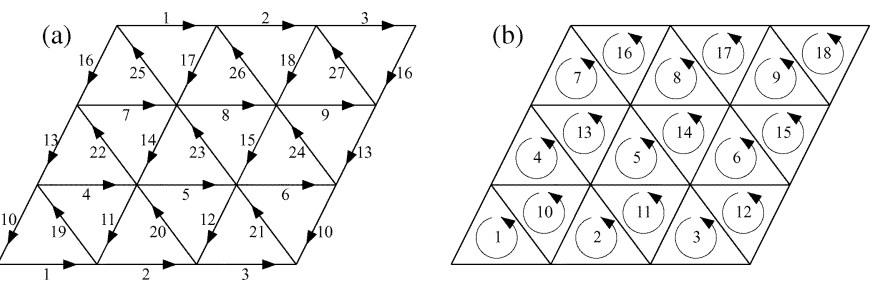

Fig. 2. Regular triangular mesh with periodic boundary condition: (a) edges and (b) faces of primal mesh.

directions for eigenvalue analysis. The permittivity $\varepsilon$ and permeability $\mu$ are assumed to be uniform everywhere.

The Whitney basis functions or piecewise uniform basis functions give $\varepsilon$ an eigenvalue $2 \beta$ or $8 \beta$, respectively, for which the eigenvector is $(1,1, \cdots, 1)^{T}$.

Reference [6] proposed an approximate method yielding $\varepsilon^{-1}$, which is constructed by assembling locally inversed $\varepsilon_{L}$. When $\varepsilon$ is approximately composed by assembling the local inversed $\varepsilon_{L}^{W}$ or $\varepsilon_{L}^{P U}$, it also has an eigenvalue $2 \beta$ or $8 \beta$, respectively, for which the eigenvector is $(1,1, \cdots, 1)^{T}$.

On the other hand, the reluctivity matrix $\boldsymbol{\nu}$ is given as

$$
\boldsymbol{\nu}=\frac{1}{\mu S} \boldsymbol{I} .
$$

\section{CFL Condition}

The matrix $\Gamma$ has an eigenvalue $\gamma$ given as

$$
\gamma= \begin{cases}\frac{3}{\beta \mu S}, & \text { (for Whitney basis function) } \\ \frac{3}{4 \beta \mu S}, & \text { (for piecewise uniform basis function) }\end{cases}
$$

for which the eigenvector is $(1,1, \cdots, 1,-1,-1, \cdots,-1)^{T}$. This is because

$$
C^{T}\left(\begin{array}{c}
1 \\
\vdots \\
1 \\
-1 \\
\vdots \\
-1
\end{array}\right)=2\left(\begin{array}{c}
1 \\
1 \\
\vdots \\
1
\end{array}\right) \quad C\left(\begin{array}{c}
1 \\
1 \\
\vdots \\
1
\end{array}\right)=3\left(\begin{array}{c}
1 \\
\vdots \\
1 \\
-1 \\
\vdots \\
-1
\end{array}\right) .
$$

Numerical eigenvalue analysis for $\Gamma$ shows that $\gamma_{\max }$ is given by $\gamma$.

For example, the eigenvalues of $\boldsymbol{\Gamma}$ are computed numerically for 2-D regular meshes consisting of $2 \times 20 \times 20$ equilateral triangle elements and isosceles right triangle elements. For simplicity, $\varepsilon$ and $\mu$ are assumed to be $1 \mathrm{~F} / \mathrm{m}$ and $1 \mathrm{H} / \mathrm{m}$, respectively. The edge length of equilateral triangle elements is $1 \mathrm{~m}$ while each of the isosceles right triangle elements has two sides of 1 $\mathrm{m}$ and a side of $\sqrt{2} \mathrm{~m}$. Fig. 3 plots the distributions of eigenvalues for the two meshes, where the eigenvalues are ordered according to their magnitudes. Fig. 3(a) also plots the eigenvalue distribution given by the diagonalized permittivity matrix for which the local matrix $\varepsilon_{L}^{D}$ becomes $\left(2 \varepsilon_{L}^{W}+\varepsilon_{L}^{P U}\right) / 3$ for equilateral triangle elements. The equilateral triangle elements give maximum eigenvalues of 48 and 12 for the Whitney and piecewise uniform basis functions, respectively. The isosceles right 
(a)
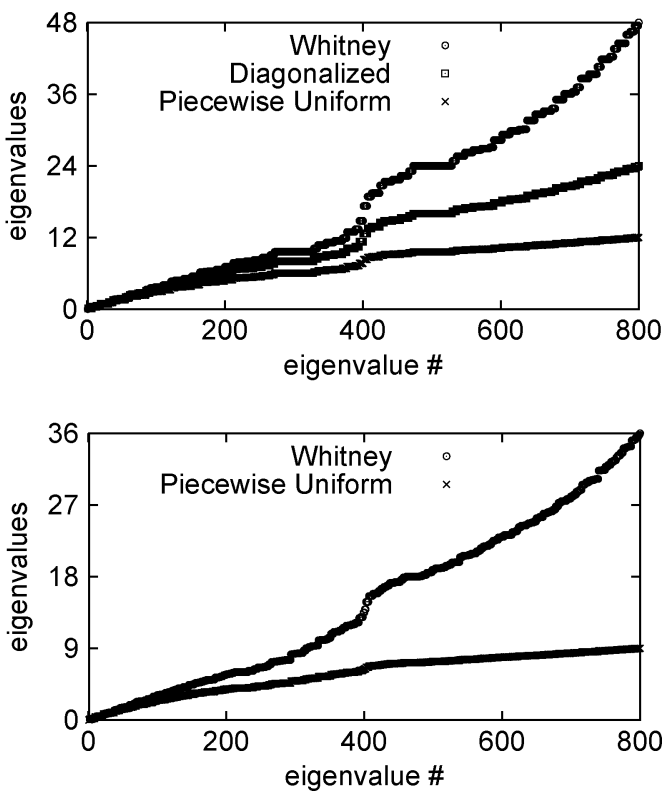

(b)

Fig. 3. Eigenvalue distribution: (a) equilateral triangle elements and (b) isosceles right triangle elements.

TABLE I

COEFFICIENT $\alpha$ IN (19) FOR THE CFL CONDITION

\begin{tabular}{|c|c|c|c|c|c|c|}
\hline element & $\mathrm{W}$ & $\mathrm{PU}$ & $\mathrm{D}$ & LI-W & LI-PU & FDTD \\
\hline \multicolumn{1}{|l}{$l, l, l$} & $\frac{1}{2 \sqrt{3}}$ & $\frac{1}{\sqrt{3}}$ & $\frac{1}{\sqrt{6}}$ & $\frac{1}{2 \sqrt{3}}$ & $\frac{1}{\sqrt{3}}$ & $\frac{1}{\sqrt{2}}$ \\
\cline { 1 - 5 }$l, l, \sqrt{2 l}$ & $\frac{1}{3}$ & $\frac{2}{3}$ & - & $\frac{1}{3}$ & $\frac{2}{3}$ & \\
\hline
\end{tabular}

triangle elements obtain maximum eigenvalues of 36 and 9. All maximum eigenvalues agree with (16).

Consequently, the CFL condition is obtained as

$\Delta t \leq \begin{cases}\frac{\sqrt{l_{1}^{2}+l_{2}^{2}+l_{3}^{2}}}{6 c}, & \text { (for Whitney basis function) } \\ \frac{\sqrt{l_{1}^{2}+l_{2}^{2}+l_{3}^{2}}}{3 c}, & \text { (for piecewise uniform basis function) }\end{cases}$

where $c=1 / \sqrt{\varepsilon \mu}$. The piecewise uniform basis functions allow a time step that is twice as large as that for the Whitney basis functions.

For example, the equilateral triangle elements and isosceles right triangle elements give the CFL condition as

$$
\Delta t \leq \frac{\alpha l}{c}
$$

where $l$ is the (shortest) edge length. The coefficient $\alpha$ is listed in Table I, where LI denotes the approximation of local inversion. The CFL condition for the diagonalized $\varepsilon$ is also shown for the equilateral triangle elements. An isosceles right triangle mesh cannot use diagonalization because its $\varepsilon_{L}$ is not strictly positive definite. The CFL condition for the finite-difference time-domain (FDTD) method is also shown for a square mesh with edge length $l$.

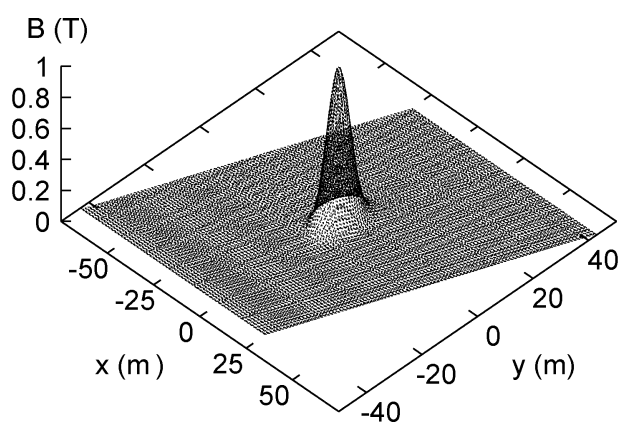

(a)

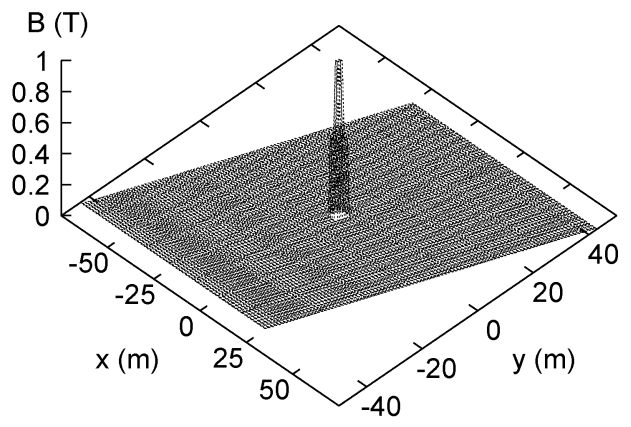

Fig. 4. Two initial conditions (a) and (b).

TABLE II

TIME STEP $\Delta(c t)$ (IN METERS)

\begin{tabular}{|c|c|c|c|c|c|c|}
\hline & $\mathrm{W}$ & $\mathrm{PU}$ & $\mathrm{D}$ & LI-W & LI-PU & FDTD \\
\hline stable & 0.28 & 0.57 & 0.4 & 0.28 & 0.57 & 0.7 \\
\hline unstable & 0.29 & 0.58 & 0.41 & 0.29 & 0.58 & 0.71 \\
\hline
\end{tabular}
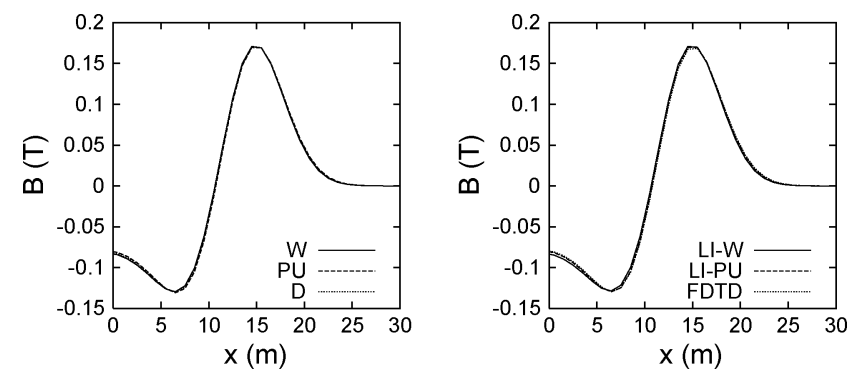

Fig. 5. Distribution of $B$ along $y=0$ at $t^{\prime}=c t=14 \mathrm{~m}$ obtained from initial condition (a).

\section{WAVE PROPAGATION COMPUTATION}

For simplicity, variables are transformed as $c t \rightarrow t^{\prime}$ and $\boldsymbol{e} / c \rightarrow \boldsymbol{e}^{\prime}$ to rewrite (1) and (2) as

$$
\begin{aligned}
\boldsymbol{e}^{k+1} & =\boldsymbol{e}^{\prime k}+\varepsilon \mu \Delta t^{\prime} \boldsymbol{\varepsilon}^{-1} \boldsymbol{C}^{T} \boldsymbol{\nu} \boldsymbol{b}^{k+1 / 2} \\
\boldsymbol{b}^{k+3 / 2} & =\boldsymbol{b}^{k+1 / 2}-\Delta t^{\prime} \boldsymbol{C} \boldsymbol{e}^{k+1}
\end{aligned}
$$

For numerical examination of the CFL condition, the regular mesh with $2 \times 100 \times 100$ equilateral triangle elements is used with periodic boundary conditions similar to the mesh in Fig. 2. The edge length of a triangle element is set to $1 \mathrm{~m}$. Two initial conditions shown in Fig. 4(a) and (b) are used with $\boldsymbol{E}=0$ at $t=0$.

Table II lists examined time steps to confirm the CFL condition, where the row "stable" gives time steps that allow stable 


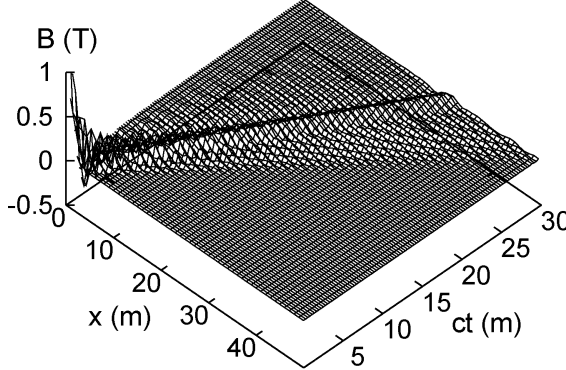

(a)

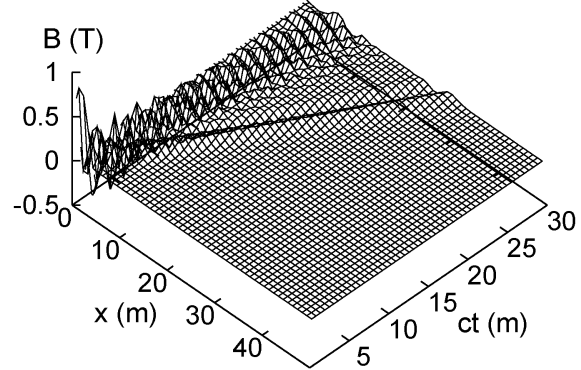

(b)

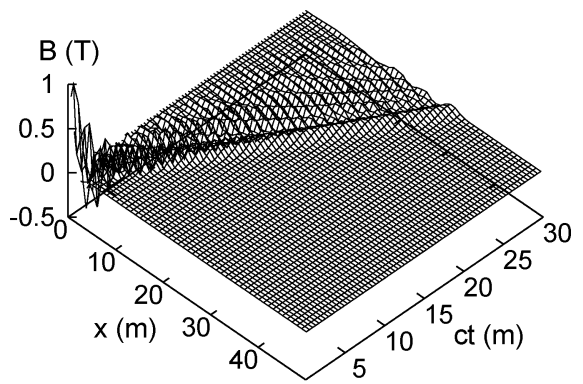

(c)

Fig. 6. Wave propagation along $y=0$ from initial condition (b): (a) with the Whitney basis functions, (b) with the piecewise uniform basis functions, and (c) with the diagonalized permittivity matrix.

computation while the row "unstable" lists time steps that result in divergence. They agree with the CFL condition shown in Table I.

Fig. 4 shows the distributions of $B$ along $y=0$ at $t^{\prime}=c t=$ $14 \mathrm{~m}$, which is obtained from the initial condition (a), where the distribution given by the FDTD scheme is also shown under a similar simulation condition for comparison. The construction of $\varepsilon$ hardly affects the distribution when the initial condition is smooth.

Fig. 5 depicts the wave propagation along $y=0$, which results from the initial condition (b). The propagation of the pulse wave is largely affected by the construction of $\varepsilon$. The Whitney basis functions yield a small wave for which the propagation speed is larger than $c$. This unphysical wave comes from a small eigenvalue $\beta$ of the local permittivity matrix. However, the unphysical wave disappears when a sufficiently small time step is used. The piecewise uniform basis functions yield an oscillation around $x=0$, which remains even if the time step is small.

\section{CONCLUDING REMARKS}

The CFL conditions of 2-D FI methods on regular triangular meshes are derived, where the Whitney basis functions and piecewise uniform basis functions are used to construct the permittivity matrix. The piecewise uniform basis functions allow a time step that is twice as large as the Whitney basis functions.

This paper compares only time-stepping stabilities of several FI methods under uniform permittivity and permeability. The accuracy of wave propagation analysis should be examined for nonuniform media.

\section{APPENDIX}

From (11), it is obvious that $\varepsilon_{L}^{P U}$ has an eigenvector $(1,1,1)$ for which the eigenvalue is $4 \beta$.

From (8), $\int_{T} \boldsymbol{w}_{1} \cdot \boldsymbol{w}_{j}(j=1,2,3)$ becomes

$$
\int_{T}\left(\boldsymbol{w}_{1}\right)^{2} d S=\frac{S}{6}\left\{\left(\nabla \lambda_{3}\right)^{2}-\nabla \lambda_{2} \cdot \nabla \lambda_{3}+\left(\nabla \lambda_{2}\right)^{2}\right\}
$$

$$
\begin{aligned}
\int_{T} \boldsymbol{w}_{1} \cdot \boldsymbol{w}_{j} d S=\frac{S}{12}\{ & \nabla \lambda_{1} \cdot \nabla \lambda_{k}-\left(\nabla \lambda_{k}\right)^{2} \\
& \left.-2 \nabla \lambda_{1} \cdot \nabla \lambda_{j}+\nabla \lambda_{j} \cdot \nabla \lambda_{k}\right\}
\end{aligned}
$$

where $(j, k)=(2,3)$ or $(3,2)$. From (9), (22), and (23)

$$
\sum_{j=1}^{3} \varepsilon_{L 1 j}^{W}=\frac{\varepsilon S}{12} \sum_{i=1}^{3}\left(\nabla \lambda_{i}\right)^{2}=\beta
$$

is obtained. Similarly, it holds that

$$
\sum_{j=1}^{3} \varepsilon_{L 2 j}^{W}=\sum_{j=1}^{3} \varepsilon_{L 3 j}^{W}=\beta
$$

From (24) and (25), $\varepsilon_{L}^{W}$ has an eigenvector $(1,1,1)$ for which the eigenvalue is $\beta$.

\section{REFERENCES}

[1] T. Weiland, "Time domain electromagnetic field computation with finite difference methods," Int. J. Numer. Model., vol. 9, pp. 295-319, 1996.

[2] I. E. Lager, E. Tonti, A. T. de Hoop, G. Mur, and M. Marrone, "Finite formulation and domain-integrated field relations in electromagnetics-A synthesis," IEEE Trans. Magn., vol. 39, pp. 1199-1202, 2003.

[3] A. Bossavit and L. Kettunen, "Yee-like schemes on a tetrahedral mesh, with diagonal lumping," Int. J. Numer. Model., vol. 12, pp. 129-142, 1999.

[4] L. Codecasa and F. Trevisan, "Piecewise uniform bases and energetic approach for discrete constitutive matrices in electromagnetic problems," Int. J. Numer. Meth. Eng., vol. 65, pp. 548-565, 2006.

[5] M. Cinalli and A. Schiavoni, "A stable and consistent generalization of the FDTD technique to nonorthogonal unstructured grids," IEEE Trans. Antennas Propag., vol. 54, no. 5, pp. 1503-1512, May 2006.

[6] P. Alotto, A. De Cian, and G. Molinari, "A time-domain 3-D fullMaxwell solver based on the cell method," IEEE Trans. Magn., vol. 42, pp. 799-802, 2006.

[7] A. Taflove and S. C. Hagness, Computational Electrodynamics: The Finite-Difference Time-Domain Method. Reading, MA: Artech House, 2005.

[8] J. F. Lee, R. Palandech, and R. Mittra, "Modeling three-dimensional discontinuities in waveguides using nonorthogonal FDTD algorithm," IEEE Trans. Microw. Theory Tech., vol. 40, pp. 346-352, Feb. 1992.

[9] R. Schuhmann and T. Weiland, "Stability of the FDTD algorithm on nonorthogonal grids related to the spatial interpolation scheme," IEEE Trans. Magn., vol. 35, pp. 2751-2754, May 1999.

[10] S. D. Gedney and J. A. Roden, "Numerical stability of nonorthogonal FDTD methods," IEEE Trans. Antennas Propag., vol. 48, pp. 231-239, Feb. 2000. 